\title{
Dynamic Hebbian learning in adaptive frequency oscillators
}

\author{
Ludovic Righetti*, Jonas Buchli, Auke Jan Ijspeert \\ School of Computer and Communication Sciences, Ecole Polytechnique Fédérale de Lausanne (EPFL), \\ IC - ISIM - Station 14, CH-1015 Lausanne, Switzerland
}

Received 8 March 2005; received in revised form 27 October 2005; accepted 10 February 2006

Communicated by J.E. Pearson

\begin{abstract}
Nonlinear oscillators are widely used in biology, physics and engineering for modeling and control. They are interesting because of their synchronization properties when coupled to other dynamical systems. In this paper, we propose a learning rule for oscillators which adapts their frequency to the frequency of any periodic or pseudo-periodic input signal. Learning is done in a dynamic way: it is part of the dynamical system and not an offline process. An interesting property of our model is that it is easily generalizable to a large class of oscillators, from phase oscillators to relaxation oscillators and strange attractors with a generic learning rule. One major feature of our learning rule is that the oscillators constructed can adapt their frequency without any signal processing or the need to specify a time window or similar free parameters. All the processing is embedded in the dynamics of the adaptive oscillator. The convergence of the learning is proved for the Hopf oscillator, then numerical experiments are carried out to explore the learning capabilities of the system. Finally, we generalize the learning rule to non-harmonic oscillators like relaxation oscillators and strange attractors.
\end{abstract}

(C) 2006 Elsevier B.V. All rights reserved.

Keywords: Adaptive frequency oscillator; Synchronization; Learning; Plasticity; Dynamical systems

\section{Introduction}

Nonlinear oscillators have been widely used to model various physical and biological processes and for the last two decades, they are also used in engineering fields, for example autonomous robotics. Models of Josephson junctions [22], lasers, central pattern generators (CPGs) [6,10, $13,23]$, associative memories $[2,18]$ and beat perception $[8,14]$ are a few examples that show the importance of oscillators in modeling and control.

Oscillator models are interesting because of their synchronization capabilities, either with other oscillators or with external driving signals. In most cases, it is a difficult task to choose the right parameters of the oscillators to ensure that they will synchronize as desired. Most studies use phase-locking behavior, but when parameters are outside the phase-locking region synchronization fails. This is mainly the case because oscillators lack plasticity, they have fixed intrinsic frequencies and cannot dynamically adapt their parameters.

\footnotetext{
* Corresponding author.

E-mail address: ludovic.righetti@a3.epfl.ch (L. Righetti).
}

Some recent studies, however, concentrate on developing dynamic plasticity for oscillators, so they can learn and synchronize with a wider range of frequencies, without one having to tune the parameters by hand $[1,2,9,15-17]$. But these attempts are so far limited to very simple classes of oscillators, equivalent to phase oscillators, mainly because this is the only class of oscillators that can be analytically studied and for which convergence can be proved, when adding adaptivity to the system. Adaptive relaxation oscillators were also developed to model rhythm perception [8]. These oscillators are able to adapt their frequencies to synchronize with external input. But these input signals are simple and reduce to periodic pulse trains.

Recently, we designed an adaptive oscillator for studying adaptive locomotion in biologically inspired robotics [3,4]. In that work we developed an adaptive frequency Hopf oscillator able to adapt to the resonant frequency of a mechanical system. The oscillator is able to adapt its frequency to the frequency of complex input signals. In this contribution, we prove the convergence of this oscillator and generalize the adaptive rule for more complex oscillators so they can learn the frequencies 
of, and synchronize with, any rhythmic input signal. An interesting property of our method is that we go beyond phaselocking of oscillations. We add plasticity to the system, in the sense that the system can change its own parameters in order to learn the frequencies of the periodic input signals. So the range of frequencies that can be learned is not limited and after learning the oscillator continues to oscillate at the learned frequency, even if the input signal disappears. We call our adaptive mechanism ${ }^{1}$ dynamic Hebbian learning because it shares similarities with correlation-based learning observed in neural networks [11].

One major aspect of our approach is that an oscillator learns the frequency of any periodic input, without any signal processing. This means that an oscillator can adapt its frequency to any kind of periodic, or even pseudo-periodic, input. The process is completely dynamic, and does not require the specification of time windows or similar free parameters as is often the case in signal processing algorithms. The whole learning process and the frequency extraction from the input is totally embedded in the dynamics of the system. Another interesting property of the method is that we can directly apply it to many kinds of oscillators, for example relaxation oscillators and strange attractors. An oscillator, perturbed by a periodic signal $F$, is described by the general equations

$\dot{x}=f(x, y, \omega)+\epsilon F$

$\dot{y}=f(x, y, \omega)$

with $\omega$ some parameter influencing the frequency of the oscillations. We introduce a learning rule for this parameter

$\dot{\omega}= \pm \epsilon F \frac{y}{\sqrt{x^{2}+y^{2}}}$.

The sign depends on the direction of rotation of the limit cycle in the $(x, y)$ phase space. This general adaptation rule works for many different oscillators, $\omega$ will converge to a value such that one frequency component of the oscillator and one of the input $F$ match. We discuss this general learning rule in this contribution.

In Section 2, we first present the adaptive learning rule with a simple Hopf oscillator and prove the convergence and the stability of the whole system. Then, in Section 3, we present some numerical simulations, to show that the oscillator can adapt its frequency to the frequency of any kind of periodic or pseudo-periodic signal. Finally, in order to demonstrate the generality of our method, we construct, in Section 4, an adaptive Van der Pol oscillator which we discuss in detail. We also present examples of frequency adaptation with an adaptive Rayleigh oscillator, an adaptive Fitzhugh-Nagumo oscillator and an adaptive Rössler system. In Section 5, we finish this contribution with a discussion.

\section{Learning frequencies with a Hopf oscillator}

In this section, we introduce the learning rule for frequency adaptation in oscillators. To keep the discussion as simple

\footnotetext{
${ }^{1}$ In this article, we use adaptation and learning as synonyms.
}

as possible, we use a Hopf oscillator to discuss our learning method, because its phase evolution is simple to describe. Generalization to more complex oscillators will be presented in further sections. We first present the model, then we prove the convergence of the adaptive dynamical system.

\subsection{Model description}

\subsubsection{The Hopf oscillator}

The dynamics of the Hopf oscillator is governed by the following differential equations

$\dot{x}=\left(\mu-r^{2}\right) x-\omega y+\epsilon F$

$\dot{y}=\left(\mu-r^{2}\right) y+\omega x$.

where $r=\sqrt{x^{2}+y^{2}}, \mu>0$ controls the amplitude of the oscillations and $\omega$ is the intrinsic frequency of the oscillator. This means that without perturbations (when $\epsilon=0$ ), the system is oscillating at $\omega \mathrm{rad} \mathrm{s}^{-1}$. This oscillator is coupled with a periodic force $F$. When the force is zero, the system has an asymptotically stable harmonic limit cycle, with radius $\sqrt{\mu}$ and frequency $\omega$. As the limit cycle of the Hopf oscillator is structurally stable, small perturbations around its limit cycle $(\epsilon>0)$ do not change the general behavior of the system. This means that the limit cycle will still exist, only its form and time scale will change. Structural stability assures that this change is close to identity.

As we are mainly interested in the phase dynamics, we rewrite the system in polar coordinates. We set $x=r \cos \phi$ and $y=r \sin \phi$. Eqs. (1) and (2) transform into

$\dot{r}=\left(\mu-r^{2}\right) r+\epsilon F \cos \phi$

$\dot{\phi}=\omega-\frac{\epsilon}{r} F \sin \phi$.

It is well known that when the oscillator has its intrinsic frequency $\omega$ close to one frequency component of the periodic input, it will phase-lock (this phenomenon is also called entrainment) [19]. This means that the oscillations synchronize with the frequency of the periodic input. The maximum distance between the intrinsic frequency of the oscillator and the periodic input that still permits phase-locking depends directly on the coupling strength. The stronger the coupling, the larger the entrainment basin. Outside this basin, the oscillator is still influenced by the coupling but does not synchronize. If the periodic input has several frequency components, then several entrainment basins will appear. Phase-locking will be possible with each frequency component. Outside the basin, the oscillator will have a tendency to accelerate or decelerate, according to the term $F \sin \phi$, on average the oscillator will tend to oscillate at a frequency which is between the intrinsic frequency of the oscillator and the frequency of the input. In the case of multi-frequency inputs, these oscillations will be influenced in a similar manner.

\subsubsection{Adaptive dynamical system}

Now we can build our adaptation rule by using the influence of the external perturbation on the activity of the oscillator. The 
adaptation rule will be a dynamical system of the form

$\dot{\omega}=f(\omega, r, \phi, F)$.

In the following we motivate the concrete choice of the adaptation rule by reasoning about the effects of a perturbation in a geometric way in the phase space of the dynamical system. This provides insights into our choice of the learning rule. In further sections, we will show more rigorously that this reasoning is appropriate and leads to the desired behavior.

To get a good grasp on the effects of perturbations on a limit cycle system (i.e. an oscillator) it is helpful to look at the limit cycle in the phase space representation. In the phase space all perturbations have a direction, i.e. they can be represented as a vector $\vec{P}$ in that space.

Due to the stability properties of a limit cycle system a perturbation can in the long term only affect the phase of the oscillator. The phase is marginally stable whereas the system is damped perpendicularly to the limit cycle. This means that the phase point always returns to the limit cycle, but it can be phase shifted. In other words the system after a singular perturbation will forget all the perturbation's influence except its influence on the phase.

Especially in a small neighborhood of the limit cycle a small perturbation can only affect the phase strongly if it perturbs the oscillator in the direction tangential to the limit cycle. The perturbations perpendicular to the limit cycle are damped out. The domain where this assumption is valid depends on the coupling of phase and radius. While for the Hopf oscillator this assumption is valid for a very large neighborhood, the neighborhood can be very small for other oscillators, e.g. oscillators with strongly bent isochrones.

To discuss the influence of the perturbation on the phase in this neighborhood, let us introduce a coordinate system with its origin on the phase point. The first base vector $\vec{e}_{r}$ is chosen perpendicular to the limit cycle, while the second base vector $\overrightarrow{e_{\phi}}$ is chosen tangential to the limit cycle (cf. Fig. 1). Thus, this coordinate system rotates with the phase point along the limit cycle. In order to find the influence $p_{\phi}=\left|\vec{p}_{\phi}\right|$ of the perturbation on the phase it is sufficient to project $\vec{P}$ on $\overrightarrow{e_{\phi}}$

$p_{\phi}=\vec{P} \cdot \overrightarrow{e_{\phi}}$.

Thus, depending on the external perturbation and the state of the oscillator (i.e. the position of the point on the limit cycle) the perturbation accelerates the phase point or slows it down. If the perturbation is a periodic signal, this results in an average acceleration or deceleration depending on the frequency difference. This effect, if the frequency of the oscillator and the external frequency are close, leads to well known phase-locking behavior. Thus, the influence carries the information needed to adjust to the frequency of the external perturbation. Consequently, if we take this same effect to tune the frequency of the oscillator (on a slower time scale) the frequency should evolve toward the frequency of the perturbation. Therefore, the effect of $f(\omega, r, \phi, F)$ on $\omega$ has to be the same as the effect of the perturbation on the phase, thus (on average) driving $\omega$ toward the frequency of the perturbation.

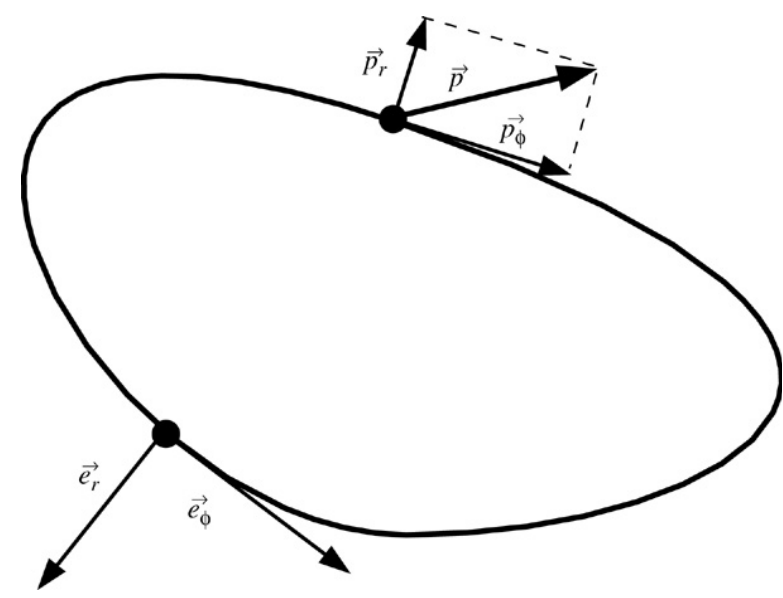

Fig. 1. We illustrate the coordinate system in which synchronization is most naturally discussed. The figure shows an arbitrary limit cycle. The system is strongly damped in the direction perpendicular to the limit cycle $\overrightarrow{e_{r}}$ and marginally stable in the direction tangential to the limit cycle $\overrightarrow{e_{\phi}}$. This is the reason for the structurally stable limit cycle in the first place and allows for a resetting of the phase on the other hand. Note that the two-dimensional representation is always valid for discussing a limit cycle since there exists always a two-dimensional manifold which contains the limit cycle. Refer to the text for a discussion of the perturbation $\vec{P}$.

While the discussion here is valid for limit cycles of any form and in any dimension, in the case of the Hopf oscillator and the perturbation as chosen in Eqs. (3) and (4) it is evident that $p_{\phi}=\frac{\epsilon}{r} F \sin \phi$. We chose accordingly

$\dot{\omega}=-\epsilon F \sin \phi$

which corresponds in Cartesian coordinates to

$\dot{\omega}=-\epsilon F \frac{y}{\sqrt{x^{2}+y^{2}}}$.

The adaptation of $\omega$ happens on a slower time scale than the evolution of the rest of the system. This adaptation time scale is influenced by the choice of $\epsilon$. Note that the $r$ variable is dropped because we do not want a learning rule which is scaled by the amplitude of the oscillations. With this rule, the oscillator will adapt to the frequency of any input signal. As in applications most signals will be non-harmonic, i.e. they have several frequency components, the oscillator will adapt to one of these components, generally the closest to the intrinsic frequency of the oscillator. We must also note that it is required to keep the oscillator coupled with the input, because it is the evolution of $\phi(t)$, i.e. change of frequency correlated with $\dot{\omega}$, that enables adaptation in Eq. (7). A proof of convergence of this adaptive oscillator (Eqs. (3), (4) and (7)) in the general case of multi-frequency inputs is given in the next section.

\subsection{Proof of convergence with the Hopf oscillator}

In this section we prove the stability of the adaptive Hopf oscillator, but we will see in following sections that the results we derive in this section can also justify convergence for other types of oscillators. The new dynamical system we study is the one composed of the oscillator and its learning rule for the frequency (Eqs. (3), (4) and (7)). As long as $\omega>0$, because of 
structural stability, the behavior of the oscillator (Eqs. (3) and (4)) is known, so we just have to prove that $\omega$ converges to the desired input frequency.

We use perturbation methods (cf. [12]) to discuss the convergence of the system. The solution of the system $\{r(t), \phi(t), \omega(t)\}$ can be written as a perturbation series, with $\epsilon<1$

$r(t)=r_{0}+\epsilon r_{1}+\epsilon^{2} r_{2}+\epsilon^{3} R_{r}$

$\phi(t)=\phi_{0}+\epsilon \phi_{1}+\epsilon^{2} \phi_{2}+\epsilon^{3} R_{\phi}$

$\omega(t)=\omega_{0}+\epsilon \omega_{1}+\epsilon^{2} \omega_{2}+\epsilon^{3} R_{\omega}$

with initial conditions $r_{0}\left(t_{0}\right)=r_{0}, \phi_{0}\left(t_{0}\right)=0$ and $\omega_{0}\left(t_{0}\right)=\omega_{0}$ independent of $\epsilon$. Here, $r_{i}, \phi_{i}$ and $\omega_{i}$ are functions of time and $R_{r}, R_{\omega}$ and $R_{\phi}$ are small residues of the order $\epsilon^{3}$, which means there exists a constant $k$ such that $R_{i}<k$, generally $k$ is small. The following proof will hold under the hypothesis that $k \ll 1$, numerical simulations in Section 3 will confirm this hypothesis. We could also expand the perturbation series to higher order: the finer the approximation, the wider the time interval valid for the approximation. But we will show that convergence appears on the time scale associated with the second order approximation. By inserting Eqs. (9)-(11) into Eqs. (3), (4) and (7), and by observing that

$$
\begin{aligned}
& \sin \left(\phi_{0}+\epsilon \phi_{1}+\epsilon^{2} \phi_{2}+\epsilon^{3} R_{\phi}\right) \\
& =\sum_{k=0}^{\infty} \frac{(-1)^{k}\left(\phi_{0}+\epsilon \phi_{1}+\epsilon^{2} \phi_{2}+\epsilon^{3} R_{\phi}\right)^{2 k+1}}{(2 k+1) !} \\
& =\sin \phi_{0}+\epsilon \phi_{1} \cos \phi_{0}+O\left(\epsilon^{2}\right)
\end{aligned}
$$

and similarly that

$$
\begin{aligned}
& \cos \left(\phi_{0}+\epsilon \phi_{1}+\epsilon^{2} \phi_{2}+\epsilon^{3} R_{\phi}\right) \\
& =\cos \left(\phi_{0}\right)-\epsilon \phi_{1} \sin \left(\phi_{0}\right)+O\left(\epsilon^{2}\right)
\end{aligned}
$$

we can identify the terms corresponding to each $\epsilon^{n}$ and derive the following differential equations

$$
\begin{aligned}
& \dot{r}_{0}=\left(\mu-r_{0}^{2}\right) r_{0} \\
& \dot{\phi}_{0}=\omega_{0} \\
& \dot{\omega}_{0}=0 \\
& \dot{r}_{1}=\mu r_{1}-3 r_{1} r_{0}^{2}+F \cos \phi_{0} \\
& \dot{\phi}_{1}=\omega_{1}-\frac{1}{r_{0}}\left(r_{1} \dot{\phi}_{0}-r_{1} \omega_{0}+F \sin \phi_{0}\right) \\
& \dot{\omega}_{1}=-F \sin \phi_{0}
\end{aligned}
$$

$\dot{r}_{2}=\mu r_{2}-3 r_{2} r_{0}^{2}-r_{2} r_{1}^{2}-F \phi_{1} \cos \phi_{0}$

$\dot{\phi}_{2}=\omega_{2}-\frac{1}{r_{0}}\left(r_{1} \dot{\phi}_{1}-r_{1} \omega_{1}+r_{2} \dot{\phi}_{0}-r_{2} \omega_{0}+F \phi_{1} \cos \phi_{0}\right)$

$\dot{\omega}_{2}=-F \phi_{1} \cos \phi_{0}$

with initial conditions $r_{0}\left(t_{0}\right)=\sqrt{\mu}, \phi_{0}\left(t_{0}\right)=0, \omega_{0}\left(t_{0}\right)=\omega_{0}$ and $r_{i}\left(t_{0}\right)=\phi_{i}\left(t_{0}\right)=\omega_{i}\left(t_{0}\right)=0, \forall i=1,2$. We consider that the unperturbed system $(i=0)$ has already converged to the limit cycle and that at time $t_{0}$, there are no perturbations. We have to solve Eqs. (16), (19) and (22) to construct an approximate solution of Eq. (7) and thus show the convergence properties of the adaptation rule $\omega$. The behavior of the two other state variables is already known since the Hopf oscillator has a structurally stable limit cycle. In order to solve these equations we also have to solve Eqs. (14), (15) and (18). The error of the approximation will be of order $O\left(\epsilon^{3}\right)$ and will hold for some time interval $\left[t_{0}, t_{0}+\sigma\right]$. The solutions of Equations Eqs. (14)-(16) are straightforward

$$
\begin{aligned}
& r_{0}(t)=\sqrt{\mu} \\
& \phi_{0}(t)=\omega_{0}\left(t-t_{0}\right) \\
& \omega_{0}(t)=\omega_{0} .
\end{aligned}
$$

To solve the other equations, we first rewrite the periodic input as its complex Fourier series

$$
F(t)=\sum_{n=-\infty}^{\infty} A_{n} \mathrm{e}^{\mathrm{i} n \omega_{F} t}
$$

Here $\omega_{F}$ is the frequency of the input. We now consider the case where $\omega_{0} \neq n \omega_{F}, \forall n \in \mathbb{N}$, which means that at the beginning the system is not synchronized with any frequency component of the periodic input $F$. We then get

$$
\begin{aligned}
\dot{\omega}_{1} & =-\left(\sum_{n=-\infty}^{\infty} A_{n} \mathrm{e}^{\mathrm{i} n \omega_{F} t}\right) \sin \left(\omega_{0}\left(t-t_{0}\right)\right) \\
& =-\sum_{n=-\infty}^{\infty} A_{n} \frac{\mathrm{e}^{\mathrm{i}\left(n \omega_{F}+\omega_{0}\right) t-\mathrm{i} \omega_{0} t_{0}}-\mathrm{e}^{\mathrm{i}\left(n \omega_{F}-\omega_{0}\right) t+\mathrm{i} \omega_{0} t_{0}}}{2 \mathrm{i}}
\end{aligned}
$$

This is solved to give

$$
\begin{aligned}
\omega_{1}(t)= & \frac{1}{2} \sum_{n=-\infty}^{\infty} A_{n}\left(\frac{-\left(\mathrm{e}^{\mathrm{i}\left(n \omega_{F}-\omega_{0}\right) t+\mathrm{i} \omega_{0} t_{0}}-\mathrm{e}^{\mathrm{i} n \omega_{F} t_{0}}\right)}{\left(n \omega_{F}-\omega_{0}\right)}\right. \\
& \left.+\frac{\left(\mathrm{e}^{\mathrm{i}\left(n \omega_{F}+\omega_{0}\right) t-\mathrm{i} \omega_{0} t_{0}}-\mathrm{e}^{\mathrm{i} n \omega_{F} t_{0}}\right)}{\left(n \omega_{F}+\omega_{0}\right)}\right)
\end{aligned}
$$

and

$\dot{\phi}_{1}=\omega_{1}+\frac{\dot{\omega}_{1}}{\sqrt{\mu}}$

which is solved to give

$$
\begin{aligned}
\phi_{1}(t)= & \frac{\omega_{1}(t)}{\sqrt{\mu}}+\frac{1}{2} \sum_{n=-\infty}^{\infty} A_{n}\left(\frac{\left(\mathrm{e}^{\mathrm{i}\left(n \omega_{F}+\omega_{0}\right) t-\mathrm{i} \omega_{0} t_{0}}-\mathrm{e}^{\mathrm{i} n \omega_{F} t_{0}}\right)}{\mathrm{i}\left(n \omega_{F}+\omega_{0}\right)^{2}}\right. \\
& +\frac{2 \omega_{0}\left(t-t_{0}\right) \mathrm{e}^{\mathrm{i} n \omega_{F} t_{0}}}{n^{2} \omega_{F}^{2}-\omega_{0}^{2}} \\
& \left.-\frac{\left(\mathrm{e}^{\mathrm{i}\left(n \omega_{F}-\omega_{0}\right) t+\mathrm{i} \omega_{0} t_{0}}-\mathrm{e}^{\mathrm{i} n \omega_{F} t_{0}}\right)}{\mathrm{i}\left(n \omega_{F}-\omega_{0}\right)^{2}}\right) .
\end{aligned}
$$

By combining Eqs. (25) and (28), we have a first order approximation $\omega(t)=\omega_{0}+\epsilon \omega_{1}(t)+\epsilon^{2} R_{\omega}$. This approximation is a periodic solution with mean equal $\omega_{0}$. Nevertheless, this first order approximation does not show any adaptation of $\omega(t)$. 
This seems normal, since we argued before that the learning takes place on a larger time scale than the perturbation (which is of order $\epsilon$ ). We now derive the second order approximation to show that learning appears on the associated time scale. As we are interested in the second order form of $\omega$, we now solve Eq. (22)

$$
\begin{aligned}
\dot{\omega}_{2}= & -\left(\sum_{m=-\infty}^{\infty} A_{m} \mathrm{e}^{\mathrm{i} m \omega_{F} t}\right)\left(\frac{\mathrm{e}^{\mathrm{i} \omega_{0}\left(t-t_{0}\right)}+\mathrm{e}^{-\mathrm{i} \omega_{0}\left(t-t_{0}\right)}}{2}\right) \phi_{1}(t) \\
= & -\frac{1}{2}\left(\sum _ { m = - \infty } ^ { \infty } A _ { m } \left(\mathrm{e}^{\mathrm{i}\left(m \omega_{F}+\omega_{0}\right) t-\mathrm{i} \omega_{0} t_{0}}\right.\right. \\
& \left.\left.+\mathrm{e}^{\mathrm{i}\left(m \omega_{F}-\omega_{0}\right) t+\mathrm{i} \omega_{0} t_{0}}\right)\right) \phi_{1}(t) .
\end{aligned}
$$

By expanding the equation we find a sum of simpler terms that can be easily integrated

$\omega_{2}=\int_{t_{0}}^{t}\left(\frac{1}{4} \sum_{m, n \in \mathbb{Z}} A_{m} A_{n}\left(E_{1}+E_{2}+E_{3}+E_{4}+E_{5}+E_{6}\right)\right)$

where

$$
\begin{aligned}
E_{1}= & \mathrm{e}^{\mathrm{i}\left((m+n) \omega_{F}+2 \omega_{0}\right) t-2 \mathrm{i} \omega_{0} t_{0}} \\
& \times\left(\frac{-1}{\sqrt{\mu}\left(n \omega_{F}+\omega_{0}\right)}-\frac{1}{\mathrm{i}\left(n \omega_{F}+\omega_{0}\right)^{2}}\right) \\
E_{2}= & \mathrm{e}^{\mathrm{i}\left((m+n) \omega_{F}-\omega_{0}\right) t+2 \mathrm{i} \omega_{0} t_{0}} \\
& \times\left(\frac{1}{\sqrt{\mu}\left(n \omega_{F}-\omega_{0}\right)}+\frac{1}{\mathrm{i}\left(n \omega_{F}-\omega_{0}\right)^{2}}\right) \\
E_{3}= & \mathrm{e}^{\mathrm{i}\left(m \omega_{F}+\omega_{0}\right) t+\mathrm{i}\left(n \omega_{F}-\omega_{0}\right) t_{0}} \\
& \times\left(\frac{-2 \omega_{0}}{\sqrt{\mu}\left(\left(n \omega_{F}\right)^{2}-\omega_{0}^{2}\right)}-\frac{4 n \omega_{F} \omega_{0}}{\mathrm{i}\left(\left(n \omega_{F}\right)^{2}-\omega_{0}^{2}\right)^{2}}\right) \\
E_{4}= & \mathrm{e}^{\mathrm{i}\left(m \omega_{F}-\omega_{0}\right) t+\mathrm{i}\left(n \omega_{F}+\omega_{0}\right) t_{0}} \\
& \times\left(\frac{-2 \omega_{0}}{\sqrt{\mu}\left(\left(n \omega_{F}\right)^{2}-\omega_{0}^{2}\right)}-\frac{4 n \omega_{F} \omega_{0}}{\mathrm{i}\left(\left(n \omega_{F}\right)^{2}-\omega_{0}^{2}\right)^{2}}\right) \\
E_{6}= & \left(\mathrm{e}^{\mathrm{i} \omega_{0}\left(t-t_{0}\right)}+\mathrm{e}^{-\mathrm{i} \omega_{0}\left(t-t_{0}\right)}\right) \\
E_{5}= & \mathrm{e}^{\mathrm{i}(m+n) \omega_{F} t}\left(\frac{-2 \omega_{0}}{\sqrt{\mu}\left(\left(n \omega_{F}\right)^{2}-\omega_{0}^{2}\right)}+\frac{4 n \omega_{F} \omega_{0}}{\mathrm{i}\left(\left(n \omega_{F}\right)^{2}-\omega_{0}^{2}\right)^{2}}\right) \\
& \times\left(m \omega_{F} t+n \omega_{F} t_{0}\right)\left(t-t_{0}\right) . \\
& \\
& \times\left(\frac{\left(n \omega_{F}^{2}-\omega_{0}^{2}\right.}{2}\right)
\end{aligned}
$$

Previously, we postulated that $\omega_{0} \neq n \omega_{F}, \forall n \in \mathbb{N}$, consequently, the integration of $E_{1}, E_{2}, E_{3}$ and $E_{4}$ gives periodic functions with zero mean. The integration of $E_{6}$ gives a function oscillating with some frequency but with its amplitude varying because of the $t$ term, the average contribution of this function is zero. The integration of $E_{5}$ is more interesting because when $n=-m$, the exponential disappears and we have a constant instead. Thus when integrating we will find linear terms. For the case $m \neq-n$, after integration, we find a periodic function with zero mean. Therefore, $\omega_{2}(t)$ is composed of a periodic function $\tilde{\omega}_{2}(t)$ with zero mean and a deviation $D_{\omega}(t)$.

$\omega_{2}(t)=\tilde{\omega}_{2}(t)+D_{\omega}(t)$

where

$$
\begin{aligned}
D_{\omega}(t)= & \int_{t_{0}}^{t} \frac{1}{4} \sum_{\substack{n \in \mathbb{Z} \\
m=-n}} A_{n} A_{m}\left(\frac{2 \omega_{0}}{\sqrt{\mu}\left(\left(n \omega_{F}\right)^{2}-\omega_{0}^{2}\right)}\right. \\
& \left.-\frac{4 n \omega_{F} \omega_{0}}{\mathrm{i}\left(\left(n \omega_{F}\right)^{2}-\omega_{0}^{2}\right)^{2}}\right) \\
= & \int_{t_{0}}^{t}\left(\frac{-A_{0}}{2 \sqrt{\mu} \omega_{0}}+\sum_{n \in \mathbb{N}^{*}} \frac{A_{n} \bar{A}_{n} \omega_{0}}{\sqrt{\mu}\left(\left(n \omega_{F}\right)^{2}-\omega_{0}^{2}\right)}\right) \\
= & \left(\frac{-A_{0}}{2 \sqrt{\mu} \omega_{0}}+\sum_{n \in \mathbb{N}^{*}} \frac{\left|A_{n}\right|^{2} \omega_{0}}{\sqrt{\mu}\left(\left(n \omega_{F}\right)^{2}-\omega_{0}^{2}\right)}\right)\left(t-t_{0}\right) .
\end{aligned}
$$

Then, the solution of $\omega(t)$ in a neighborhood of $t_{0}$ is

$$
\omega(t)=\omega_{0}+\epsilon \omega_{1}(t)+\epsilon^{2} \tilde{\omega}_{2}(t)+\epsilon^{2} D_{\omega}(t)+O\left(\epsilon^{3}\right) .
$$

The solution is composed of small oscillations of amplitude much smaller than $\epsilon$ around $\omega_{0}$ and a slight deviation $\epsilon^{2} D_{\omega}(t)$. This deviation term determines how the frequency converges to the input frequency. It can also be used to predict the basins of attraction for inputs with several frequency components (cf. Section 3.3). For an input signal that has only one frequency in its spectrum, the deviation is obviously in the direction of this frequency, since $D_{\omega}(t)>0$ when $\omega_{F}>\omega_{0}$ and $D_{\omega}(t)<0$ otherwise. As this approximation is valid for any $\omega_{0}$ and any $t_{0}$, i.e. the point in time when we make the approximation is not important, the oscillator will always, on average, change its frequency in the direction of the input frequency. For more complex signals with more than one frequency component, because of the $\left(n \omega_{F}\right)^{2}-\omega_{0}^{2}$ term in $D_{\omega}$, the system will just change its frequency according to the distance between its intrinsic frequency $\omega_{0}$ and the frequency components of the input. The amplitudes $A_{n}$ of the frequency components will also influence this convergence, in the sense that the more intensity a frequency component has, the more it will attract $\omega(t)$. Section 3 shows examples of such convergence. We must also note that the zero frequency (the mean of the periodic signal) can also influence the convergence because of the $A_{0}$ term. Thus, if the input signal has a non-zero mean, $\omega$ could eventually converge to 0 if $A_{0}$ has a stronger influence than the other frequency components. In this case, the limit cycle of the Hopf oscillator would bifurcate into a fixed point.

We still have to discuss the case $\omega_{0}=n \omega_{F}$ for a given $n \in \mathbb{N}$. In this case, the oscillator is synchronized with one frequency component of the perturbation. Thus, $\omega(t)$ oscillates and deviates from $n \omega_{F}$. Then there are two cases, either the deviation becomes an attraction as soon as $\omega_{0} \neq n \omega_{F}$ and the 
intrinsic frequency of the oscillator is always staying in a small neighborhood of $n \omega_{F}$, or $\omega(t)$ diverges from this frequency and gets attracted by another frequency component of the input signal, with stronger amplitude.

We notice that $\epsilon$ controls both the amplitude of oscillations around $n \omega_{F}$ and the learning rate of the system (proportional to $\epsilon^{2}$ ). So the faster the learning is, the higher the error of adaptation will be. But as $\epsilon<1$, the error of adaptation is bounded and small (of the order of $\epsilon$ ).

So we have proved that the learning rule makes the frequency converge to a frequency component of the input signal, for any initial conditions $\left(t_{0}, \omega_{0}\right)$. The attracting frequency component depends on its distance to the intrinsic frequency of the oscillator and its intensity. The proof is global because we did not make any assumption on the initial condition for $\omega$ and on the neighborhood of the attracting frequencies.

\section{Numerical simulations}

The goal of this section is to study the behavior of the learning dynamical system with numerical simulations. First we give a simple example of adaptation of the oscillator receiving a simple periodic signal as input. Then we confirm the proof of Section 2.2 by calculating the second order approximation error for a simple example. We also use the analytic results to predict the behavior of the system when varying several parameters. Finally, we show that the system can adapt to pseudo-periodic signals.

\subsection{Simple example of learning}

First of all, we want to show a simple example of how the system works and discuss the influence of the learning rate $\epsilon$. The adaptive Hopf oscillator is composed of the perturbed Hopf oscillator

$\dot{x}=\left(\mu-r^{2}\right) x-\omega y+\epsilon F$

$\dot{y}=\left(\mu-r^{2}\right) y+\omega x$

and of the adaptive frequency learning rule

$\dot{\omega}=-\epsilon F \frac{y}{\sqrt{x^{2}+y^{2}}}$.

Here we use a simple cosine signal $F=\cos (30 t)$ as input, with $\mu=1$ and initial conditions $r(0)=1, \phi(0)=0$ and $\omega(0)=40$. We integrate the system numerically for several values of $\epsilon$, the results of the simulations are shown in Fig. 2. In this figure, we can see that the oscillator adapts its intrinsic frequency to the frequency of the input signal. We also see that $\epsilon$ controls the adaptation rate of the system, the higher $\epsilon$ is, the faster the learning.

\subsection{Error evaluation of the analytic approximation for a simple perturbing force}

In Section 2.2, we derived an approximate solution of the learning dynamical system, in order to prove its convergence.

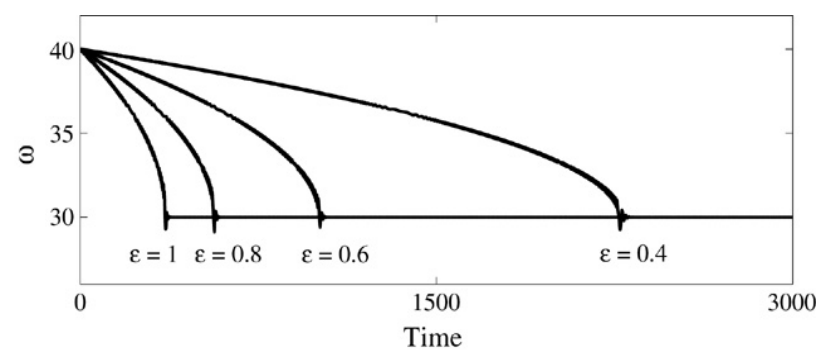

Fig. 2. Plot of the evolution of $\omega$ for four different values of $\epsilon$. Here we set $\mu=1, x(0)=1$ and $y(0)=0$, the perturbing force is $F=\cos (30 t)$. For every value of $\epsilon$, we see that $\omega$ converges to 30, which is the frequency of the input signal. Therefore, the system is able to learn the frequency of the input signal. We also notice that $\epsilon$ controls the convergence rate, the higher it is, the faster the system learns.

The error of this approximation is bounded by some constant $k$. We now evaluate numerically the error of the approximation, for a simple sinusoidal input, in order to show that this constant is really small and that the hypothesis made for proving convergence holds. We set $F=\sin \left(\omega_{F} t\right), t_{0}=0, \mu=1$. Then we can derive an approximate solution of $\omega(t)$ using Eqs. (25), (28) and (32).

$$
\begin{aligned}
& \omega_{0}(t)=\omega_{0} \\
& \omega_{1}(t)=-\frac{1}{2\left(\omega_{F}-\omega_{0}\right)} \sin \left(\left(\omega_{F}-\omega_{0}\right) t\right) \\
& +\frac{1}{2\left(\omega_{F}+\omega_{0}\right)} \sin \left(\left(\omega_{F}+\omega_{0}\right) t\right) \\
& \omega_{2}(t)=\frac{\sin \left(2 \omega_{0} t\right)}{16 \omega_{0}\left(\omega_{F}-\omega_{0}\right)}-\frac{\sin \left(2 \omega_{F} t\right)}{16 \omega_{F}\left(\omega_{F}-\omega_{0}\right)} \\
& -\frac{\sin \left(2\left(\omega_{F}-\omega_{0}\right) t\right)}{16\left(\omega_{F}-\omega_{0}\right)^{2}}+\frac{t}{8\left(\omega_{F}-\omega_{0}\right)} \\
& -\frac{t}{8\left(\omega_{F}+\omega_{0}\right)}+\frac{\sin \left(2\left(\omega_{F}+\omega_{0}\right) t\right)}{16\left(\omega_{F}+\omega_{0}\right)^{2}} \\
& -\frac{\sin \left(2 \omega_{0} t\right)}{16 \omega_{0}\left(\omega_{F}+\omega_{0}\right)}+\frac{\sin \left(2 \omega_{F} t\right)}{16 \omega_{F}\left(\omega_{F}+\omega_{0}\right)} \\
& +\frac{\cos \left(2 \omega_{F} t\right)-1}{16 \omega_{F}\left(\omega_{F}-\omega_{0}\right)^{2}}+\frac{\cos \left(2 \omega_{0} t\right)-1}{16 \omega_{0}\left(\omega_{F}-\omega_{0}\right)^{2}} \\
& +\frac{\cos \left(2\left(\omega_{F}-\omega_{0}\right) t\right)-1}{16\left(\omega_{F}-\omega_{0}\right)^{3}} \\
& -\frac{\cos \left(\left(\omega_{F}+\omega_{0}\right) t\right)-1}{4\left(\omega_{F}-\omega_{0}\right)^{2}\left(\omega_{F}+\omega_{0}\right)} \\
& -\frac{\cos \left(\left(\omega_{F}-\omega_{0}\right) t\right)-1}{4\left(\omega_{F}-\omega_{0}\right)^{3}}-\frac{\cos \left(2\left(\omega_{F}+\omega_{0}\right) t\right)-1}{16\left(\omega_{F}+\omega_{0}\right)^{3}} \\
& -\frac{\cos \left(2 \omega_{F} t\right)-1}{16 \omega_{F}\left(\omega_{F}+\omega_{0}\right)^{2}}+\frac{\cos \left(2 \omega_{0} t\right)-1}{16 \omega_{0}\left(\omega_{F}+\omega_{0}\right)^{2}} \\
& +\frac{\cos \left(\left(\omega_{F}+\omega_{0}\right) t\right)-1}{4\left(\omega_{F}+\omega_{0}\right)^{3}} \\
& -\frac{\cos \left(\left(\omega_{F}-\omega_{0}\right) t\right)-1}{4\left(\omega_{F}+\omega_{0}\right)^{2}\left(\omega_{F}-\omega_{0}\right)} \text {. }
\end{aligned}
$$

We can now numerically evaluate the errors of the approximations of order $1, \omega_{\epsilon}(t)=\omega_{0}+\epsilon \omega_{1}(t)$, and of order 

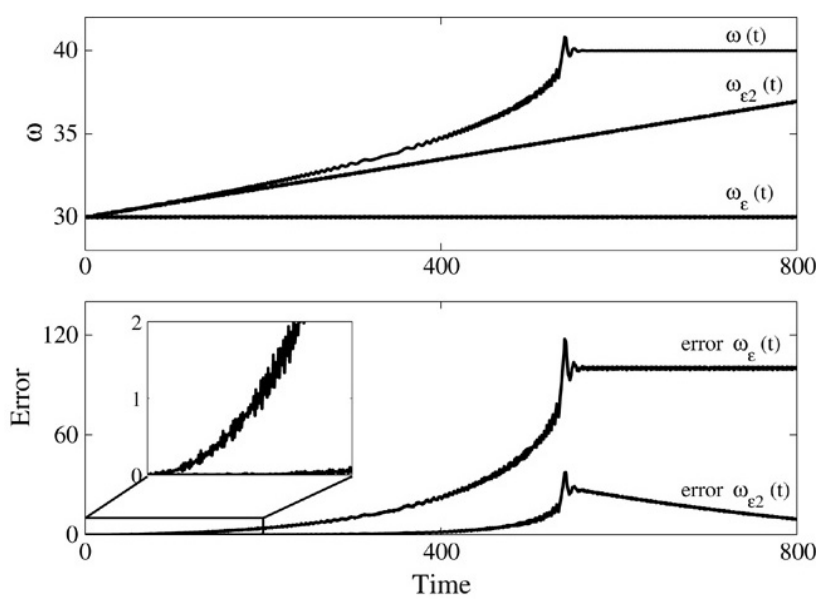

Fig. 3. Results of the simulation of the first and second order approximations. For a simple input, here $F=\sin (40 t), \epsilon=0.9$, initial conditions are $t_{0}=0$, $w_{0}=30$. The upper figure shows the evolution of the $\omega$ variable for the initial dynamical system (Eq. (38)), the first order approximation $\omega_{\epsilon}(t)$ and the second order approximation $\omega_{\epsilon^{2}}(t)$. The lower figure shows quadratic errors between the initial system and the two approximations, for the evolution of $\omega$.

2, $\omega_{\epsilon^{2}}(t)=\omega_{0}+\epsilon \omega_{1}(t)+\epsilon^{2} \omega_{2}(t)$. The upper plot of Fig. 3 shows the result of this simulation. First of all, we clearly see that the dynamical system correctly learns the frequency of the input signal. In this figure we also plotted the functions $\omega_{\epsilon}(t)$ and $\omega_{\epsilon^{2}}(t)$, we clearly see that the second order approximation is really better than the first and explains the behavior of the system on a larger time scale. Actually, it explains very well the convergence process of the learning dynamical system. We see that the learning appears on a coarser time scale than the oscillations of the system. In the lower plot, we see the square error between the original system and the approximations. We clearly see that the second order approximation follows the real system for quite a long time. Table 1 summarizes the maximum square error of the approximations. It must be noted that numerical integration of the dynamical system is done with an embedded Runge-Kutta-Fehlberg $(4,5)$ algorithm, with absolute and relative errors of $10^{-6}$. As a matter of fact, errors below this value cannot be taken as significant errors. Obviously, the first order approximation diverges rapidly, at $0.1 \mathrm{~s}$ of simulation, the error is becoming really significant. On the other hand, the second order approximation is really good still after $10 \mathrm{~s}$. These results validate the hypothesis of the approximation methods and so, the analytic proof. This also emphasizes the fact that learning takes place on a larger time scale than the perturbations on the oscillator and its oscillations. Consequently, the adaptive Hopf oscillator has two distinct time scales. The finer one describes the perturbation on the oscillator and its oscillations. Learning takes place on the coarser one.

\subsection{Predicting learning with multi-frequency inputs}

When learning the frequency of multi-frequency input signals, we might expect the system to converge to one of the frequency components of the input. But how can we calculate the range of initial frequencies for which the adaptive oscillator will converge to a specific frequency component of the input?
Table 1

This table summarizes the maximum errors of the simulation for the first and second order approximations discussed from Fig. 3

\begin{tabular}{lll}
\hline Time $(\mathrm{s})$ & Maximum error $\omega_{\epsilon}$ & Maximum error $\omega_{\epsilon}{ }^{2}$ \\
\hline 0 & 0 & 0 \\
0.001 & $5.18 \mathrm{e}^{-13}$ & $1.70 \mathrm{e}^{-19}$ \\
0.01 & $4.91 \mathrm{e}^{-7}$ & $1.15 \mathrm{e}^{-12}$ \\
0.1 & 0.0053 & $6.30 \mathrm{e}^{-11}$ \\
1 & 0.0114 & $1.85 \mathrm{e}^{-7}$ \\
10 & 0.0340 & $4.25 \mathrm{e}^{-4}$ \\
\hline
\end{tabular}

While proving the convergence of the system, we derived a deviation equation, Eq. (34), that describes the deviation from the initial intrinsic frequency, $\omega_{0}$, of the oscillator

$D_{\omega}(t)=\left(\frac{-A_{0}}{2 \sqrt{\mu} \omega_{0}}+\sum_{n \in \mathbb{N}} \frac{\left|A_{n}\right|^{2} \omega_{0}}{\sqrt{\mu}\left(\left(n \omega_{F}\right)^{2}-\omega_{0}^{2}\right)}\right)\left(t-t_{0}\right)$.

We saw that this equation depends on the initial frequency of the system $\omega_{0}$, the frequency components of the periodic input $n \omega_{F}$ and their amplitude $A_{n}$. Thus, for a given input signal, we can calculate the values of $\omega_{0}$ for which the function is equal to zero $\forall t$. These zeros give the intervals of convergence, the dynamical system converging towards the frequency components located in the same interval as $\omega_{0}$.

For example consider the following input

$F=0.2 \sin (20 t)+0.5 \sin (30 t)+0.3 \sin (40 t)$.

The main frequency of this signal is $\omega_{F}=10$. The amplitude of the frequency component are $A_{2}=\frac{0.2}{2 \mathrm{i}}, A_{3}=\frac{0.5}{2 \mathrm{i}}, A_{4}=\frac{0.3}{2 \mathrm{i}}$ and $A_{i}=0, \forall i \in \mathbb{N} \backslash\{2,3,4\}$. Thus we only have to find the roots of the following equation

$\frac{0.2^{2} \omega_{0}}{4\left(20^{2}-\omega_{0}^{2}\right)}+\frac{0.5^{2} \omega_{0}}{4\left(30^{2}-\omega_{0}^{2}\right)}+\frac{0.3^{2} \omega_{0}}{4\left(40^{2}-\omega_{0}^{2}\right)}=0$.

The solutions of this equation are 0 and $\pm \sqrt{\frac{717 \pm \sqrt{134089}}{0.76}}$. As we are working with frequencies $>0$ we have the following bounds $\omega_{\text {down }} \simeq 21.3598$ and $\omega_{\text {up }} \simeq 37.8233$. Thus we must expect to have convergence to 20,30 or 40 when $\omega_{0} \in\left[0, \omega_{\text {down }}\right]$, $\left[\omega_{\text {down }}, \omega_{\text {up }}\right],\left[\omega_{\text {up }}, \infty\right]$ respectively, with some uncertainty at the limit of the intervals, because of the oscillations of order $\epsilon$ that can make the system switch from one interval to the other. Fig. 4 shows this behavior, the horizontal dotted lines mark the bounds. Convergence corresponds to what we predicted.

\subsection{Learning the pseudo-period of chaotic signals}

We proved convergence for periodic signals, but we argue that even pseudo-periodic signals can be used as input for the learning dynamical system. In order to show this fact, we present the result of learning, when coupled to a chaotic pseudoperiodic signal. We couple the oscillator with the $z$ variable of the Lorenz system [21], whose equation is

$\dot{x}=-\sigma x+\sigma y$

$\dot{y}=-x z+r x-y$

$\dot{z}=x y-b z$. 




Fig. 4. In this figure, we plotted $\omega(t)$ for several initial conditions, $\omega_{0}$. The periodic input is Eq. (43), $\epsilon=0.9$. The dotted lines indicate the boundary between the different basins of attraction, corresponding to the different frequency components of the input, that were predicted analytically.
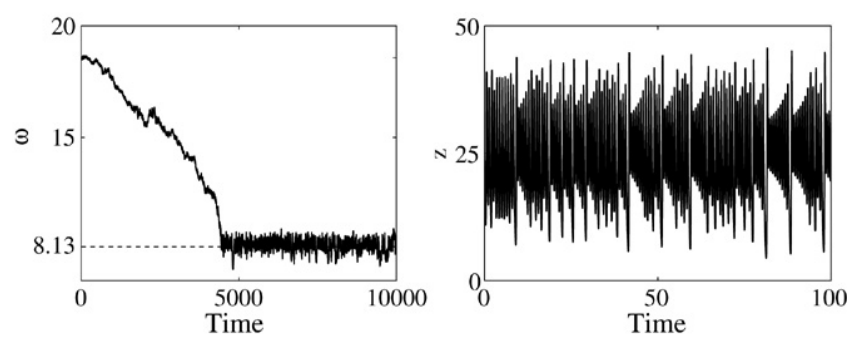

Fig. 5. The left plot of this figure represents the evolution of $\omega(t)$ when the adaptive Hopf oscillator is coupled to the $z$ variable of the Lorenz attractor. The right plot represents the $z$ variable of the Lorenz attractor. We clearly see that the adaptive Hopf oscillators can correctly learn the pseudo-frequency of the Lorenz attractor. See the text for more details.

Here $\sigma=10, r=28$ and $b=\frac{8}{3}$ (parameters for which the system produces a strange attractor). The Fourier spectrum of the $z$ variable indicates two major frequency components (data not shown), the first one at frequency $0\left(A_{0}\right.$ in the Fourier series), because the average of $z,\langle z\rangle \neq 0$, and the second one at $\sim 1.3 \mathrm{~Hz}$. As the zero-frequency component has a really strong amplitude compared to the other and we do not want adaptation to this frequency, we center the $z$ variable before coupling to the oscillator. Otherwise, $\omega$ converges to 0 and the oscillations disappear. Indeed the basin of attraction corresponding to frequency $\sim 1.3 \mathrm{~Hz}$ is not very wide and $\omega$ gets kicked out of it because of the chaotic nature of the input. Thus the input for coupling we use is $F=z-\langle z\rangle$.

Fig. 5 shows the result of the learning process. After convergence, $\langle\omega\rangle \simeq 8.13 \mathrm{rad} \mathrm{s}^{-1}$ which corresponds to an intrinsic frequency of the oscillator of $\sim 1.29 \mathrm{~Hz}$. Thus our adaptive dynamical system has learned the pseudo-frequency of the strange attractor. As this is not a strictly periodic signal, $\omega(t)$ oscillates, following the constantly changing pseudofrequency of the attractor.

This experiment enforces the idea that our adaptive dynamical system is able to learn the frequency of any periodic, or pseudo-periodic signal. It learns a frequency component of the input, even if the signal is really noisy or if the frequency is not strictly defined.

\section{Generalization to non-harmonic oscillators}

In previous sections, we presented an adaptive Hopf oscillator able to learn the frequency component of a periodic signal. The goal of this section is to show how we can easily apply our adaptive rule to non-harmonic oscillators like relaxation oscillators. The problem with such oscillators is that they have two time scales (slow buildup and fast relaxation) so it is difficult to treat them analytically to prove convergence of the adaptive rule. In this section, we discuss in detail the case of the Van der Pol oscillator, then we show results for the adaptive rule with the Rayleigh oscillator, the Fitzhugh-Nagumo oscillator and the Rössler system.

\subsection{An adaptive Van der Pol oscillator}

\subsubsection{The Van der Pol oscillator}

The Van der Pol is a classical example of relaxation oscillator and is often used in biological modeling, for example to model CPGs for quadrupedal locomotion [5]. Its equation is

$\ddot{x}+\alpha\left(x^{2}-p^{2}\right) \dot{x}+\omega^{2} x=0$.

Here $\alpha$ controls the degree of nonlinearity of the system (the relaxation part), $p$ the amplitude of the oscillations and $\omega$ mainly influences the frequency of the oscillations. In this study we set the amplitude of oscillations to $p=1$. We rewrite the system in a two-dimensional form and perturb it in the direction of $x$ as we did in Section 2

$\dot{x}=y+\epsilon F$

$\dot{y}=-\alpha\left(x^{2}-1\right) y-\omega^{2} x$.

Because of the relaxation property of the oscillator, the frequency spectrum contains, in addition to the frequency of the oscillations, an infinite number of frequency components. They are all multiples of the frequency of the oscillations and have smaller intensities. The nonlinear part of the system, whose importance is driven by the $\alpha$ variable, influences the intensity of these components. This means the higher $\alpha$ is, the more intensity high frequency components have. The frequencies of the oscillations are mainly defined by $\omega$, but $\alpha$ also influences this frequency. In fact an increase of the nonlinear term $\alpha$ tends to slow the oscillator down.

Fig. 6 shows the frequency spectrum of the $x$ variable for two different values of $\alpha$. We clearly see that the intensities of the fast frequency components increase as $\alpha$ increases. We also observe that the oscillator gets slower when $\alpha$ increases (the peaks shift to the left). But still $\omega$ is a good control parameter for the frequency of the system.

The complexity of the frequency spectrum of such oscillators complicates learning. Indeed, according to the initial conditions (i.e. according to the distance between the frequency of the periodic force and the main frequency of the oscillator), 

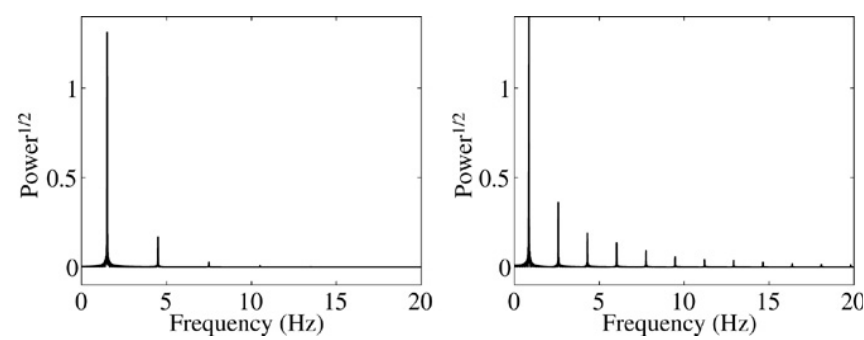

Fig. 6. Frequency spectra of the Van der Pol oscillator, both plotted with $\omega=10$. The left figure is an oscillator with $\alpha=10$ and on the right the nonlinearity is higher, $\alpha=50$. On the $y$-axis we plotted the square root of the power intensity, in order to be able to see smaller frequency components.

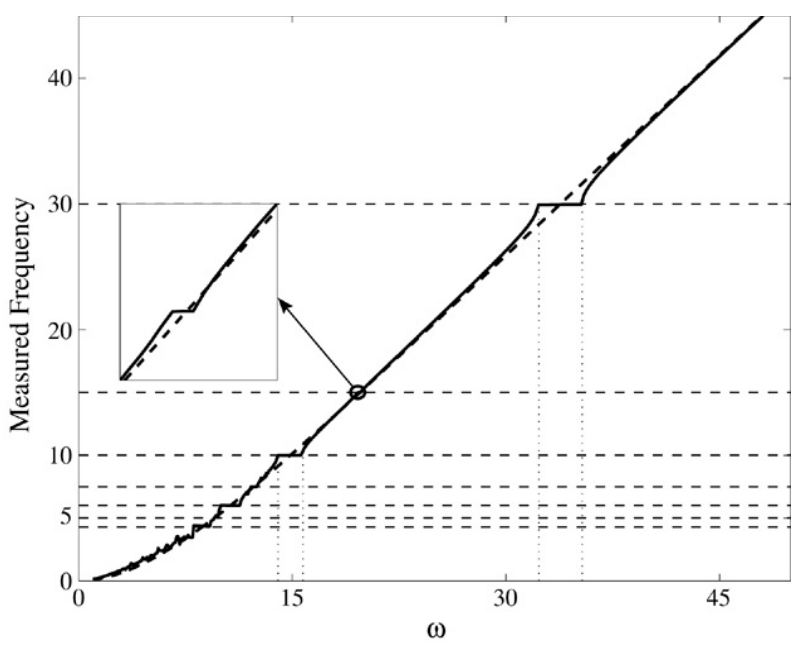

Fig. 7. Plots of the frequency of the oscillations of the Van der Pol oscillator according to $\omega$. Here $\alpha=50$. There are two plots, for the dotted line the oscillator is not coupled and for the plain line the oscillator is coupled to $F=\sin 30 t$. The strength of coupling is $\epsilon=2$. We clearly see basins of phaselocking, the main one for frequency of oscillations 30 . The other major basins appear each $\frac{30}{n}$ (dotted horizontal lines). We also notice small entrainment basins for some frequencies of the form $\frac{30 p}{q}$. For a more detailed discussion of these results refer to the text.

the oscillator may learn different frequencies and synchronize one of its higher frequency components to the input, instead of adapting its main frequency.

\subsubsection{The adaptive dynamical system}

The adaptive rule we introduced in this article dynamically changes the parameter that mainly controls the frequency of the oscillations. Thus, in this case we will make the $\omega$ parameter a dynamical system. Before discussing adaptation, we want to discuss the locations of the entrainment basins as a function of $\omega$, in order to understand how the adaptive rule will work. The entrainment basins are the regions of frequencies where the oscillator phase-locks with an input signal [19].

Fig. 7 shows the entrainment basins of a Van der Pol oscillator with high nonlinear component $\alpha=50$, which is forced by a periodic signal $\sin (30 t)$. As expected, we see phaselocking at frequency of oscillations 30, with an entrainment basin of $\omega \in[32,35]$. We also explained that the oscillator may phase-lock its higher frequency components, as these frequency components are equally spaced, one should expect phase-lock for fractions of the frequency of the perturbing force. In this case, for example, we see phase-locking at frequencies of oscillations $\frac{30}{2}, \frac{30}{3}$ and $\frac{30}{4}$.

This figure may become even more complex if the input signal has several frequency components. We would see entrainment basins every time a frequency component of the oscillator is close enough to any frequency component of the external signal. Then, when using our adaptive rule, one should expect convergence to any entrainment basins, depending on the initial conditions. Therefore, the oscillator might adapt its higher frequency components to the frequency of the input.

We now discuss the learning rule we introduced in Section 2, applied to the Van der Pol oscillator. We just change the sign of Eq. (7). This is justified because when looking to the limit cycle of the Van der Pol oscillator, we see that it is rotating in the opposite direction to the Hopf oscillator limit cycle. So the learning rule is

$\dot{\omega}=\epsilon F \frac{y}{\sqrt{x^{2}+y^{2}}}$.

We do not give an analytical proof of convergence for the Van der Pol oscillator because to use perturbation methods, as we did for the Hopf oscillator, we need to know the solution for the unperturbed Van der Pol oscillator, but to the best of our knowledge, only implicit solutions are known [7] and thus such a proof is beyond the scope of this article. But the general behavior of the system should be qualitatively the same, because of the linear coupling on the oscillator. Let us rewrite Eqs. (49) and (50) into polar coordinates

$$
\begin{aligned}
& \dot{r}=\epsilon F \cos \phi+\left(1-\omega^{2}\right) r \cos \phi \sin \phi+\alpha r^{3} \sin ^{4} \phi \\
& \dot{\phi}=-\omega^{2} \cos ^{2} \phi-\sin ^{2} \phi+\alpha r^{2} \sin ^{3} \phi \cos \phi-\frac{\epsilon F}{r} \sin \phi .
\end{aligned}
$$

Even if the phase evolution is more complex than for the Hopf oscillator, the interaction between the phase of the oscillator $\phi$ and the perturbation $F$ is of the same kind. Indeed, we clearly identify the same $-\frac{\epsilon F}{r} \sin \phi$ terms for the phase for both oscillators (Eqs. (52) and (53) and Eqs. (3) and (4)). So we can expect the same deviation of $\omega$ and therefore, the same convergence properties.

Now that we have discussed the different expected behaviors, we present a series of experiments in order to confirm our predictions and the functionality of the adaptive dynamical system.

\subsubsection{Numerical confirmation}

We predicted that the adaptive Van der Pol oscillator will either adapt its frequency of oscillations or one of its higher frequency components to the frequency of the input. In order to show this, we study the convergence of $\omega$ for different initial conditions, when the oscillator is coupled with a simple sinusoidal input $(F=\sin (30 t))$. Fig. 8 shows the result of the simulation.

When the initial condition $\omega_{0}>23$, we clearly see that $\omega$ converges to 34 which corresponds to a frequency of 


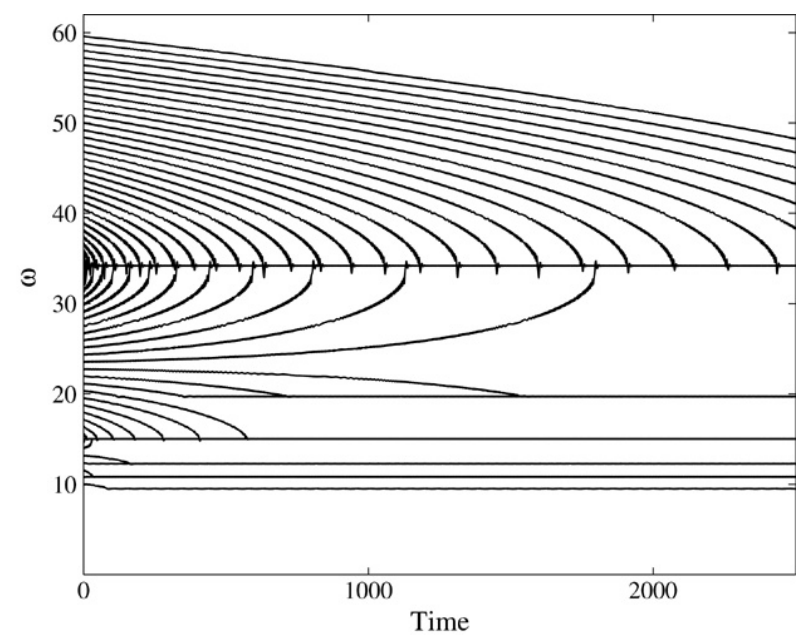

Fig. 8. This figure shows the convergence of $\omega$ for several initial frequencies. The Van der Pol oscillator is perturbed by $F=\sin (30 t)$, with coupling $\epsilon=0.7$, $\alpha=50$. We clearly see that the convergence directly depends on the initial conditions and as expected the different kinds of convergence correspond to the several entrainment basins of Fig. 7.

oscillations of $30 \mathrm{rad} \mathrm{s}^{-1}$. In this case the oscillator is correctly adapting its frequency to the frequency of the input. For lower values of $\omega_{0}$, we see convergence to other frequencies, corresponding to the entrainment basins of Fig. 7. We can conclude that the adaptive rule is changing $\omega$ in order to get one frequency component of the oscillator to the same frequency as the input signal. In fact, $\omega$ is falling into the nearest entrainment basin. Therefore, we see how useful entrainment basin studies are for understanding the dynamics of the adaptive oscillator.

Moreover, even if there is not a direct relation between $\omega$ and the frequency of the oscillations, the adaptive learning rule can appropriately tune $\omega$ so that the frequency of oscillations (or one of the other frequencies of the oscillator) is the same as the frequency of the input signal. Fig. 9 shows the result of the adaptation of the oscillator for various input signals. From these experiments, we see that $\omega$ converges to a value that corresponds to a correct adaptation of the frequency of the oscillations to the frequency of the input. In each experiment, we see that after learning, the Van der Pol oscillator and the input signal are oscillating at the same frequency.

The adaptive Van der Pol oscillator demonstrates how to generalize our adaptive rule to complex oscillators. But, an increase in the complexity of the frequency spectrum of an oscillator also generates side effects, like adaptation toward synchronization of the higher frequency components of the oscillator and the frequency of an input signal. Thus, when using highly nonlinear oscillators, one should always know the kind of frequency spectrum it has, in order to be able to predict the behavior of the oscillator. Even if we cannot analytically prove the convergence of our model, by numerically calculating the positions of the entrainment basins of the oscillator when perturbed, we are able to predict the behavior of the system in a quite powerful way.

In this section, we also discussed a very important property of the adaptive learning rule. Although the parameter we tune does not have a linear relation with the frequency of the oscillator, as is often the case for highly nonlinear oscillators, the adaptive oscillator is able to correctly adapt this parameter and find the appropriate frequency of oscillations. It seems that a monotone relation between the frequency of the oscillations and the parameter we tune is sufficient for frequency adaptation.

\subsection{Other examples of adaptive oscillators}

In this section, in order to show the generality of the adaptive rule, we present experimental results with three other oscillators. We build an adaptive Rayleigh oscillator, an adaptive Fitzhugh-Nagumo oscillator and an adaptive Rössler system.

The construction of the adaptive dynamical system is straightforward. The main task is to identify in each oscillator the parameter that most influences the frequency of the oscillations. Then, we only have to make this parameter a dynamical system in the same way as we did for the Hopf or the Van der Pol oscillator. The right column of Fig. 10 gives the resulting equations for each oscillator.

In order to demonstrate the frequency adaptivity of these modified oscillators, we made experiments for each oscillator. The results of the experiments are summarized in Fig. 10. In these experiments, the oscillators were perturbed by a simple sinusoidal input and each oscillator was able to adapt its $\omega$ parameter in order to learn the frequency of the input. Moreover, although the parameters controlling the frequency in each oscillator are not linearly related to the frequency of the oscillations, the adaptive rule is able to correctly find the correct value for the $\omega$ parameter to learn the desired frequency.

\section{Discussion}

Fields such as control of autonomous robots and signal processing may need models of plastic dynamical systems to adapt to a constantly changing environment. Moreover, plasticity in nonlinear oscillators might become an important aspect in modeling adaptive processes, for example in biology where adaptivity and memory are major properties of living systems. The learning rule presented in this article is a step towards a general framework of plastic dynamical systems, which are systems for which learning is embedded in their dynamics and not an offline optimization process.

The evolution of the parameter controlling the frequency of the adaptive oscillators that we discussed can be viewed as the correlation between the phase of the oscillator and the input signal. So we defined a type of correlation-based learning for periodic functions. In neurobiology, correlationbased learning rules are known as Hebbian learning [11], hence we call our rule dynamic Hebbian learning to highlight its correlation properties. The possible relevance to biology has to be investigated in further research.

The construction of adaptive oscillators that we presented is simple, and general enough to be applied to non-harmonic oscillators and not only to phase oscillators. The adaptive rule is general for an oscillator, perturbed by a signal $F(t)$, with general equation 
(a)
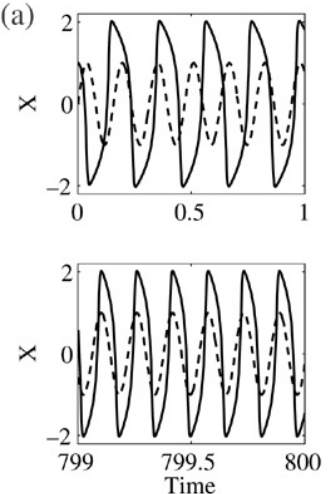

(c)
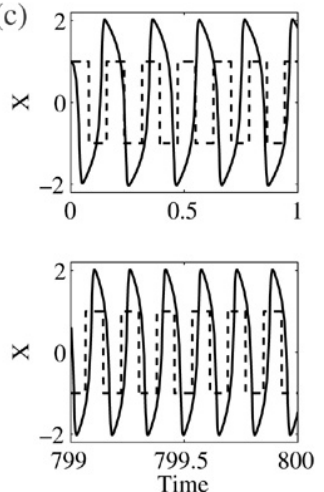
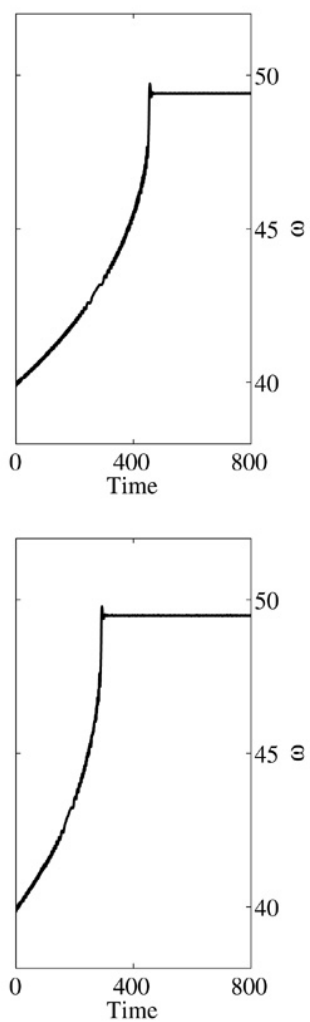

(b)
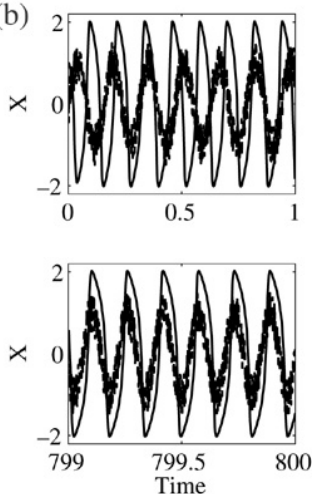

(d)
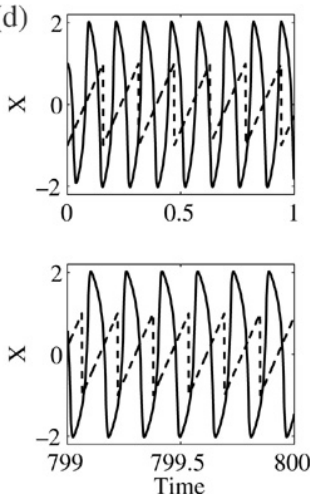
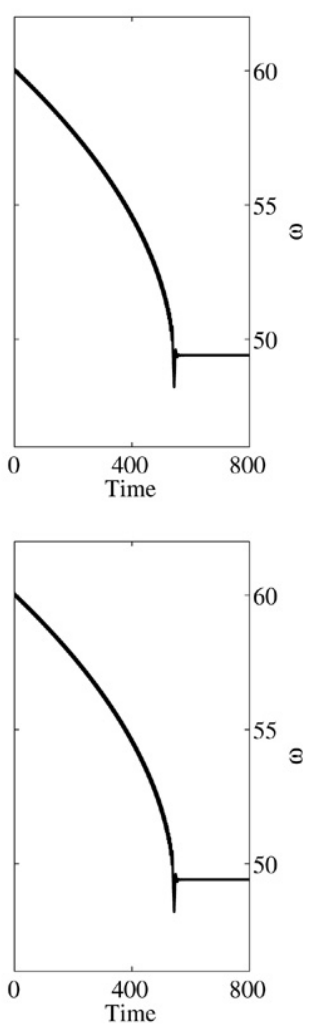

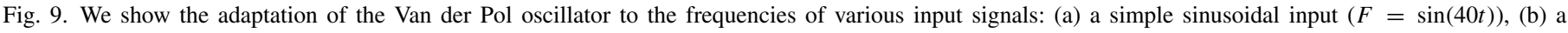

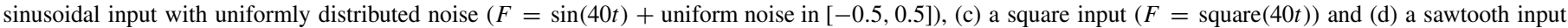

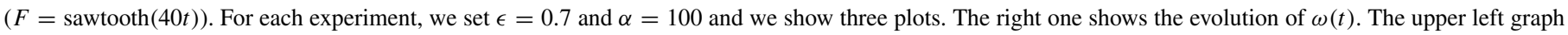



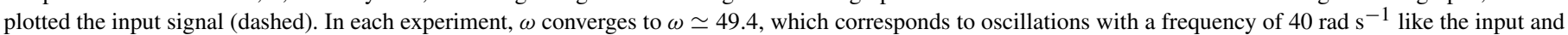
thus the oscillator correctly adapts its frequency to the frequency of the input.

$\dot{x}=f(x, y, \omega)+\epsilon F(t)$

$\dot{y}=f(x, y, \omega)$

with $\omega$ influencing the frequency of the oscillations. We have the general learning rule

$\dot{\omega}=-\epsilon F \frac{y}{\sqrt{x^{2}+y^{2}}}$.

Only the sign in front of $F$ may change according to the orientation of the flow of the oscillator in the phase space. In this sense we generalize the concept of learning presented by Nishii in [16,17], in which learning rules were only derived for phase oscillators. Nevertheless, in addition to frequency adaptation, Nishii also derived learning rules for coupling strength in populations of oscillators, which is an issue we do not address in this contribution.

The learning rule we presented is not rigid and can be modified. For instance, for the Hopf oscillator, a change in the learning rule in Eq. (7), from $\sin \phi$ to $\cos \phi$ or any combination of periodic functions will not change the convergence properties. This would only correlate the force with more complex periodic functions instead of $\sin \phi$. Intuitively, the proof of convergence should give the same results, since the learning part of the approximation (Eq. (34)) depends on the conjugate symmetry of the complex Fourier series of the input signal, which is true for every real input signal.
The mathematical proof given in this paper leads to a better comprehension of the learning process, which takes place on a coarser time scale than the oscillations of the system. This proof also allows us to predict what the oscillator would learn in the case of multi-frequency inputs. Nevertheless, we only give a proof for the adaptive Hopf oscillator and even if we numerically show that more complex adaptive oscillators can be designed, a general rigorous proof for a larger class of oscillators is still missing. Constructing such a proof is a very difficult task.

A major feature of our learning rule is that the oscillator can extract the frequency of any input signal without any explicit signal processing (Fourier transform) or any explicit time window or similar parameters. All the processing is embedded in the dynamics of the oscillator. We also showed that the system can learn frequencies from really noisy signals or from pseudo-periodic signals, like a signal from the Lorenz strange attractor. The adaptive rule is also valid for tuning parameters that do not control linearly the frequency of the oscillations. A monotonic, possibly nonlinear, relation between the frequency of oscillations and the adapted parameter is sufficient for correct adaptation of the parameter as we showed for the case of relaxation oscillators. In this case, the system is able to correctly find a value that produces oscillations at the same frequency as the input signal. 


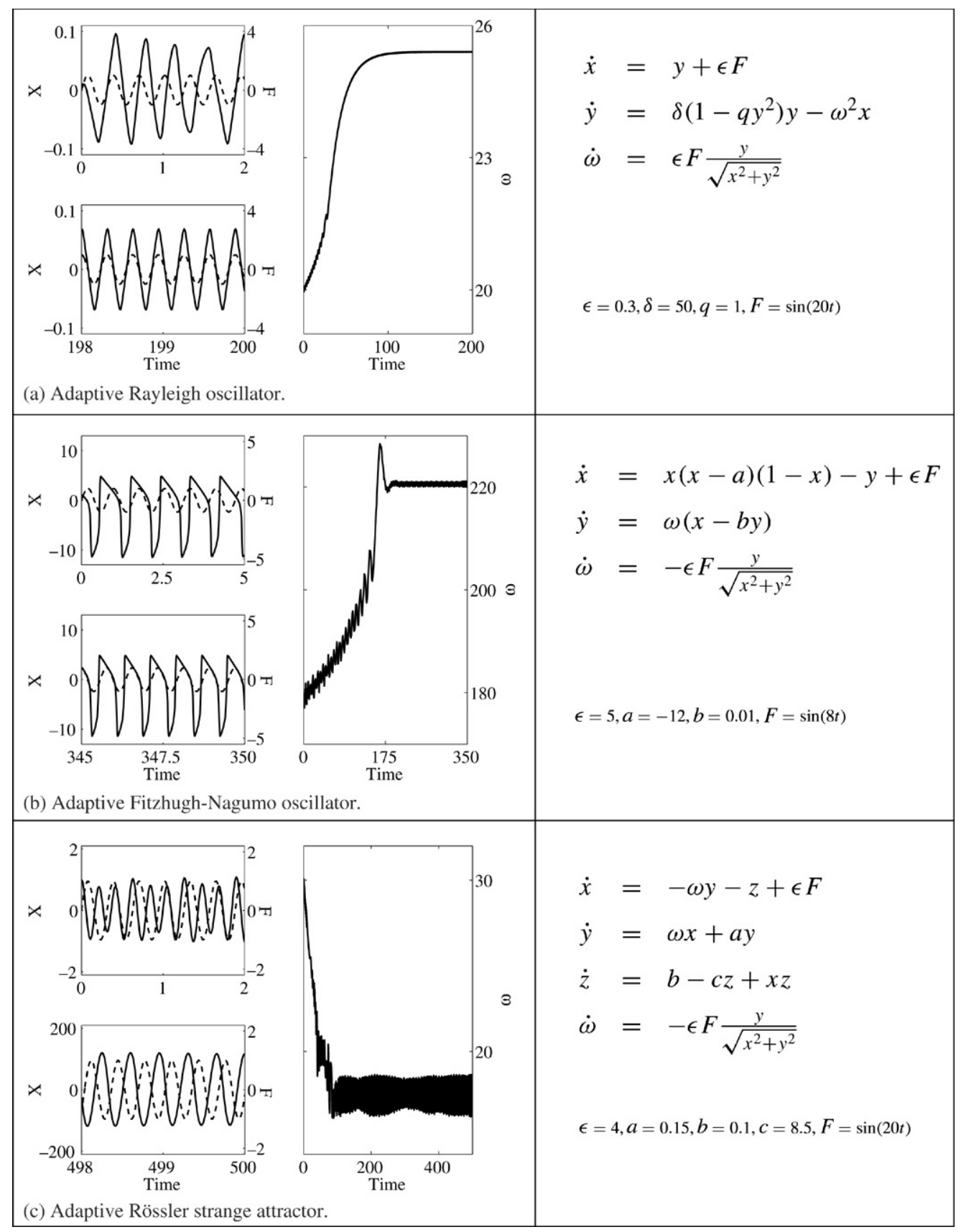

Fig. 10. We show results for several adaptive oscillators. For each oscillator, we give its equation in the right column, $\omega$ corresponding to the adaptive parameter. We also specify the values of the different parameters used in the experiments. In the left column we plotted results of the experiment. Each figure is composed of three plots. The right one is a plot of the evolution of $\omega$. The left ones are plots of the oscillations (the $x$ variable) and of the input signal $F$ (dashed line), before (upper figure) and after (lower figure) adaptation.

Dynamic Hebbian learning for adaptive oscillators has an important implication in the design of CPG models. Actually, coupled nonlinear oscillators are often used for modeling CPGs $[6,10,13,23]$, but the coupling has to be defined by hand and this is a non-trivial task. By using adaptive oscillators, one could build CPGs that can dynamically adapt their frequencies and consequently, create a desired pattern of oscillations. For instance, we are currently exploring how a population of adaptive oscillators can implement some kind of dynamic Fourier transform [20]. Furthermore, one can imagine using this adaptation mechanism to model various processes where selfsynchronization is observed.

\section{Acknowledgements}

We would like to gratefully acknowledge Jun Nakanishi and Biljana Petreska for their useful comments on a previous version of this article and the anonymous reviewers for their constructive comments. This work was made possible thanks to the support of the European Commission (Integrated Project RobotCub) and of the Swiss National Science Foundation (Young Professorship Award to Auke Ijspeert). 


\section{References}

[1] J.A. Acebron, R. Spigler, Adaptive frequency model for phase-frequency synchronization in large populations of globally coupled nonlinear oscillators, Phys. Rev. Lett. 81 (1998) 2229-2232.

[2] R. Borisyuk, M. Denham, F. Hoppensteadt, Y. Kazanovich, O. Vinogradova, Oscillatory model of novelty detection, Network: Comput. Neural Syst. 12 (2001) 1-20.

[3] J. Buchli, A.J. Ijspeert, A simple, adaptive locomotion toy-system, in: S. Schaal, A.J. Ijspeert, A. Billard, S. Vijayakumar, J. Hallam, J.A. Meyer (Eds.), From Animals to Animats 8. Proceedings of the Eighth International Conference on the Simulation of Adaptive Behavior, SAB'04, MIT Press, 2004, pp. 153-162.

[4] J. Buchli, L. Righetti, A.J. Ijspeert, A dynamical systems approach to learning: a frequency-adaptive hopper robot, in: M.S. Capcarrere, A.A. Freitas, P.J. Bentley, C.G. Johnson, J. Timmis (Eds.), Proceedings of the VIIIth European Conference on Artificial Life, ECAL 2005, in: Lecture Notes in Artificial Intelligence, vol. 3630, Springer Verlag, Berlin, Heidelberg, 2005, pp. 210-220.

[5] J.J. Collins, S.A. Richmond, Hard-wired central pattern generators for quadrupedal locomotion, Biol. Cybern. 71 (1994) 375-385.

[6] J.J. Collins, I. Stewart, Hexapodal gaits and coupled nonlinear oscillator models, Biol. Cybern. 68 (1993) 287-298.

[7] J.M. Dixon, J.A. Tuszyński, D. Sept, Orthogonal trajectories and analytical solutions of the Van der Pol equation without forcing, Phys. Lett. A 239 (1998) 65-71.

[8] D. Eck, Finding downbeats with a relaxation oscillator, Psychol. Res. 66 (2002) 18-25.

[9] B. Ermentrout, An adaptive model for synchrony in the firefly Pteroptyx malaccae, J. Math. Biol. 29 (1991) 571-585.

[10] M. Golubitsky, I. Stewart, P.-L. Buono, J.J. Collins, A modular network for legged locomotion, Physica D 115 (1998) 56-72.
[11] R. Kempter, W. Gerstner, J.L. van Hemmen, Hebbian learning and spiking neurons, Phys. Rev. E 59 (1999) 4498-4514.

[12] H.K. Khalil, Nonlinear Systems, Prentice-Hall, 1996.

[13] N. Kopell, G.B. Ermentrout, Coupled oscillators and the design of central pattern generators, Math. Biosci. 90 (1988) 87-109.

[14] E.W. Large, J.F. Kolen, Resonance and the perception of musical meter, Connection Sci. 6 (1994) 177-208.

[15] J. Nakanishi, J. Morimoto, G. Endo, G. Cheng, S. Schaal, M. Kawato, Learning from demonstration and adaptation of locomotion with dynamical movement primitives, Robot. Auton. Syst. 47 (2003) 79-91.

[16] J. Nishii, A learning model for oscillatory networks, Neural Netw. 11 (1998) 249-257.

[17] J. Nishii, Learning model for coupled neural oscillators, Network: Comput. Neural Syst. 10 (1999) 213-226.

[18] T. Nishikawa, F.C. Hoppensteadt, Y.C. Lai, Oscillatory associative memory network with perfect retrieval, Physica D 197 (2004) 134-148.

[19] A. Pikovsky, M. Rosenblum, J. Kurths, Synchronization, A universal concept in nonlinear sciences, in: Cambridge Nonlinear Science Series, vol. 12, Cambridge University Press, Cambridge, UK, 2001.

[20] L. Righetti, J. Buchli, A.J. Ijspeert, From dynamic Hebbian learning for oscillators to adaptive central pattern generators. in: Proceedings of $3 \mathrm{rd}$ International Symposium on Adaptive Motion in Animals and Machines, AMAM 2005, Verlag ISLE, Ilmenau, 2005. Full paper on CD.

[21] S. Strogatz, Nonlinear Dynamics and Chaos. With applications to Physics, Biology, Chemistry, and Engineering, Addison-Wesley Publishing Company, 1994.

[22] S.H. Strogatz, R.E. Mirollo, Splay states in globally coupled Josephson arrays: analytical prediction of Floquet multipliers, Phys. Rev. E 47 (1) (1993) 220-227.

[23] G. Taga, Y. Yamagushi, H. Shimizu, Self-organized control of bipedal locomotion by neural oscillators in unpredictable environment, Biol. Cybern. 65 (1991) 147-159. 